九州大学学術情報リポジトリ

Kyushu University Institutional Repository

\title{
An Empirical Study on Influence of Approximation Approaches on Enhancing Fireworks Algorithm
}

Pei, Yan

Graduate School of Design, Kyushu University

Zheng, Shaogiu

Peking University

Tan, Ying

Peking University

Takagi, Hideyuki

Faculty of Design, Kyushu University

http://hdl. handle. net/2324/1905845

出版情報: Proceedings 2012 IEEE International Conference on Systems, Man, and Cybernetics (SMC), pp. 1322-1327, 2012-10-17. IEEE

バージョン :

権利関係 : 


\title{
An Empirical Study on Influence of Approximation Approaches on Enhancing Fireworks Algorithm
}

\author{
Yan Pei*, Shaoqiu Zheng ${ }^{\dagger}$, Ying Tan $^{\dagger}$ and Hideyuki Takagi ${ }^{\ddagger}$ \\ *Graduate School of Design, Kyushu University, Fukuoka, Japan 815-8540 \\ Email: peiyan@kyudai.jp \\ $\dagger$ Department of Machine Intelligence, School of Electronics Engineering and Computer Science, Peking University \\ Key Laboratory of Machine Perception (Ministry of Education), Peking University, Beijing,100871, P.R. China \\ Email: zhengshaoqiu@pku.edu.cn,ytan@pku.edu.cn \\ ${ }^{\ddagger}$ Faculty of Design, Kyushu University, Fukuoka, Japan 815-8540 \\ Homepage: http://www.design.kyushu-u.ac.jp/ takagi/
}

\begin{abstract}
This paper presents an empirical study on the influence of approximation approaches on accelerating the fireworks algorithm search by elite strategy. In this study, we use three sampling data methods to approximate fitness landscape, i.e. the best fitness sampling method, the sampling distance near the best fitness individual sampling method and the random sampling method. For each approximation methods, we conduct a series of combinative evaluations with the different sampling method and sampling number for accelerating fireworks algorithm. The experimental evaluations on benchmark functions show that this elite strategy can enhance the fireworks algorithm search capability effectively. We also analyze and discuss the related issues on the influence of approximation model, sampling method, and sampling number on the fireworks algorithm acceleration performance.
\end{abstract}

Index Terms-Fireworks Algorithm, Elite Strategy, Fitness Landscape, Approximation, Acceleration

\section{INTRODUCTION}

Evolutionary computation (EC) acceleration is a promising study direction in recent EC community [6]. On the one hand, the acceleration approach study is practical fundamental to establish the EC convergence theory, on the other hand, accelerated EC algorithms (ECs) benefit their practical applications in industrial society. Approximating ECs fitness landscape and conducting an elite strategy is one of the effective approaches to accelerate ECs, Reference [11] proposed an approximation approach in original $n$-D dimensional search space to accelerate EC and References [5] and [7] extended its work to approximate fitness landscape in projected one dimensional search space.

Fireworks algorithm is a new ECs and a population based optimization technique [12]. Compared with the other ECs, fireworks algorithm presents a different search manner, which is to search the nearby space by simulating the explosion phenomenon. Since it was proposed, fireworks algorithm has shown its significance and superiority in dealing with the optimization problems of non-negative matrix factorization [1] [2]. Although the fireworks algorithm has shown its advantage than the other EC algorithms, it still has the further improvement possibility by elite strategy. To obtain elite from approximated fitness landscape, in this paper, we used some variations of sampling methods for approximating fireworks algorithm fitness landscape. It includes the best fitness sampling method, the sampling distance near the best fitness individual sampling method and the random sampling method. We apply an elite strategy to enhance fireworks algorithm search capability with different sampling methods and different sampling data number.

The originalities of this paper are: (1). This study discovers that elite strategy is an efficient method to accelerate the fireworks algorithm search; (2). We investigate the influence of sampling methods on acceleration performance of fireworks algorithm; (3). We also investigate the influence of sampling number on acceleration performance of the fireworks algorithm in each sampling method.

Following this introductory section, we conduct an overview of the fireworks algorithm in section II. Section III presents the approach design to accelerate fireworks algorithm and some new approximation methods are applied to approximate fitness landscape. To investigate and verify the performance of the proposed algorithm, a series of comparative evaluations are conducted by ten benchmark functions in section IV. In section $\mathrm{V}$, we discuss some related topics on the influence of sampling method and sampling data number on the approximation accuracy and acceleration performance, other related issues are also involved. Finally, we conclude the whole study and present the future works in section VI.

\section{FIREWORKS AlgORITHM}

\section{A. Philosophy of Fireworks Algorithm}

The fireworks algorithm generates offspring candidates around their parent individual in search space as if the fireworks explosion generates sparks around its explosion point. Given a single objective function $f: \Omega \subseteq R^{n} \rightarrow R$, it is supposed that fireworks algorithm is to find a point $x \in \Omega$, which has the minimal fitness. It is assumed that the population size of fireworks is $N$ and the population size of generated spark is $\hat{\mathrm{M}}$. Each fireworks $i(i=1,2, \ldots N)$ in a population has the following properties: a current position $x_{i}$, a current explosion amplitude $A_{i}$ and the number of generated spark 
$s_{i}$. The fireworks generates a number of sparks within a fixed explosion amplitude. AS to the optimization problem $f$, a point with better fitness is considered as a potential solution, which the optima locate nearby with high chance, vice versa.

\section{B. Spark Number and Explosion Amplitude}

For defining these properties, the fireworks algorithm makes the simulations of fireworks explosion to generate a number of sparks $s_{i}$ within the explosion amplitude $A_{i}$ by Equations (1) and (2), where $y_{\max }=\max \left(f\left(x_{i}\right)\right)$ and $y_{\min }=\min \left(f\left(x_{i}\right)\right)$, $i=1,2, \ldots N$. Here $\hat{A}$ and $M$ are constants which are denoted by a practical optimization problem.

$$
\begin{aligned}
& s_{i}=M \cdot \frac{y_{\max }-f\left(x_{i}\right)+\varepsilon}{\sum_{i=1}^{n}\left(y_{\max }-f\left(x_{i}\right)\right)+\varepsilon} \\
& A_{i}=\hat{A} \cdot \frac{f\left(x_{i}\right)-y_{\min }+\varepsilon}{\sum_{i=1}^{n}\left(f\left(x_{i}\right)-y_{\min }\right)+\varepsilon}
\end{aligned}
$$

\section{Spark Generative Operations}

In each generation, $N$ fireworks set off within a feasible bounds within explosion amplitude $A_{i}$ and spark size $s_{i}$, then the spark are generated by Equation (3). In addition, the fireworks algorithm also takes Gaussian mutation operators (Equation (4)) to enhance local search capability.

$$
\begin{gathered}
x_{i}^{j}=x_{i}^{j}+A_{i} * \operatorname{rand}(-1,1) \\
x_{i}^{j}=x_{i}^{j} * \operatorname{Gaussian}(1,1)
\end{gathered}
$$

The best firework is kept for the next generation, and the other $N-1$ fireworks for the next generation are selected based on their distance to other fireworks or randomly as to keep the diversity in the set, which includes the $N$ fireworks, the generated spark and Gaussian mutation fireworks. The fireworks algorithm continues conducting these operations till the termination criteria is satisfied.

\section{Fireworks Algorithm Acceleration by Elite STRATEGY}

\section{A. Motivation}

It is a promising study topic on approximating fitness landscape to accelerate ECs search, Reference [3] investigated the fitness landscape approximation approaches and recent related topic on surrogate-assisted EC was reported in Reference [4]. A novel fitness landscape approximation method using Fourier Transform was introduced in [8] [9]. References [5], [7], and [11] conducted some related works on fitness landscape approximation for accelerating ECs in $n$-D and 1-D dimensional search space, respectively. However, previous works show little concern on the influence of the different sampling methods with the different number to the ECs approximation model accuracy and acceleration performance.

Some related research topics need to be investigated furthermore. Firstly, there is no related report on the different sampling method influence to the approximation model accuracy and fireworks algorithm acceleration. In this study, we use three sampling data, i.e. best sampling, distance near the best fitness individual sampling method and random sampling to investigate the sampling method influence to the model accuracy and fireworks algorithm acceleration. Secondly, we also want to investigate the influence of different sampling number on the approximation model accuracy and fireworks algorithm acceleration. In this work, we set the different sampling number $(3,5,10)$ in each sampling method to conduct a set of comparative evaluations to study on those issues.

\section{B. Sampling Methods}

1) Best Sampling Method: selects the best $K$ individuals as sampling data.

2) Distance Near the Best Fitness Individual Sampling Method: selects the nearest $K$ individuals to the best individual using Euclidean distance as sampling data.

3) Random Sampling Method: selects $K$ individuals randomly as sampling data.

\section{Fireworks Algorithm with an Elite Strategy}

Our elite strategy for approximating fitness landscape uses only one of the $n$ parameter axes at a time instead of all $n$ parameter axes, and projects individuals onto each 1-D regression space. Each of the $n 1-\mathrm{D}$ regression spaces has $M$ projected individuals, which come from the different sampling methods with a different sampling number. We approximate the landscape of each 1-D regression space using the projected $M$ individuals and select the elite from the $n$ approximated 1-D landscape shapes.

In our evaluations, the sampling number is set as 3,5 and 10 ; i.e. $K=3, K=5$ and $K=10$ to check the sampling number's influence to the acceleration performance. We test least squares approximation for approximating the 1-D regression search spaces with one and two degree polynomial functions. The elite are generated from the resulting approximated shapes, see Fig. 1.

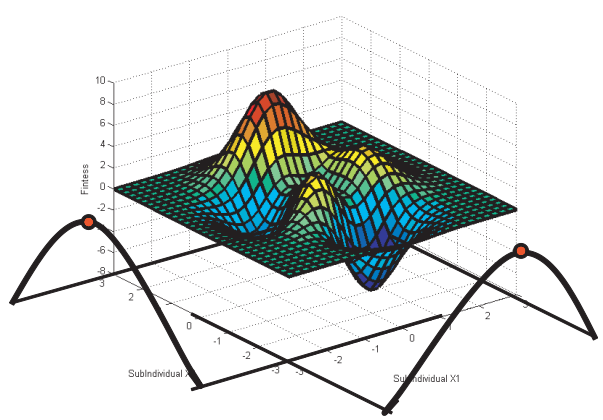

Fig. 1. Original $n$-D space and 1-D spaces obtained by reducing the dimensions of the original one.

The actual least square regression functions used is polynomial curve fitting, given by the Equations (5). 


$$
\left(\begin{array}{cccc}
x_{11}^{t} & x_{12}^{t-1} & \ldots & x_{1 K}^{0} \\
x_{21}^{t} & x_{21}^{t-1} & \ldots & x_{2 K}^{0} \\
\vdots & \vdots & & \vdots \\
x_{D 1}^{t} & x_{D 2}^{t-1} & \ldots & x_{D K}^{0}
\end{array}\right)\left(\begin{array}{c}
a_{1} \\
a_{2} \\
\vdots \\
a_{K}
\end{array}\right)=\left(\begin{array}{l}
y_{1} \\
y_{2} \\
\vdots \\
y_{K}
\end{array}\right)
$$

where $x_{i j},(i=1,2, \ldots, D)$ and $(j=1,2, \ldots, K)$ are the projected individual of point set $X_{i},(i=1,2, \ldots, D)$ and their fitness values among $\left(x_{i j}, y_{j}\right)$ in the $i$-th 1-D regression space for $(i=1,2, \ldots, D)$ and $(j=1,2, . ., K), a_{0}, a_{1}, \cdots, a_{k}$ are the parameters obtained by least squares method, $t$ is the power of polynomial function.

Least square approximation by two degree polynomial function (LS2) simplifies a regression space with a nonlinear curve, and it is easy to obtain its inflection point from its gradient, using the inflection point as the elite. Linear least square approximation (LS1) uses a linear function to approximate the regression space. Its gradient is either descent or ascent. A safer approach, taking into account both descent and ascent, is to select the average point of the linear approximation line as the elite.

The proposed methods replace the worst individual in each generation with the selected elite. Although we cannot deny the small possibility that the global optimum is located near the worst individual, the possibility that the worst individual will become a parent in the next generation is also low; removing the worst individual therefore presents the least risk and is a reasonable choice.

The whole flowing of fireworks algorithm with an elite strategy shows in Algorithm 1.

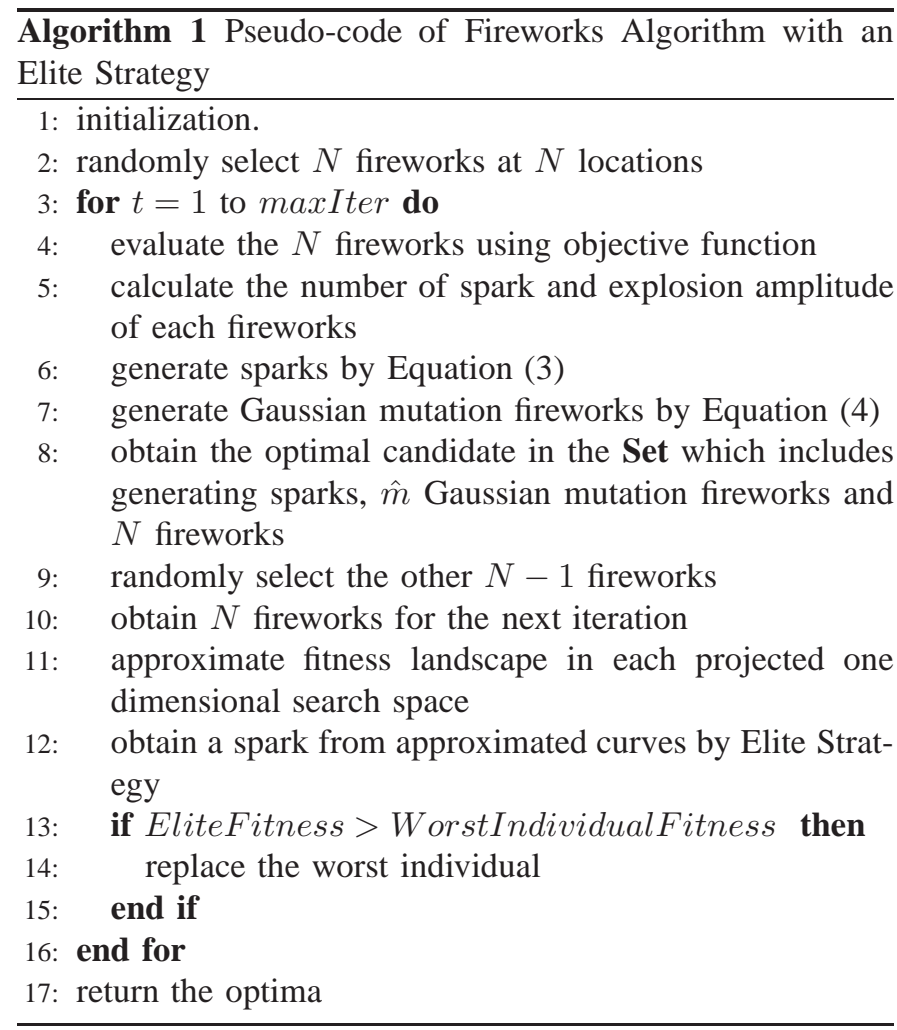

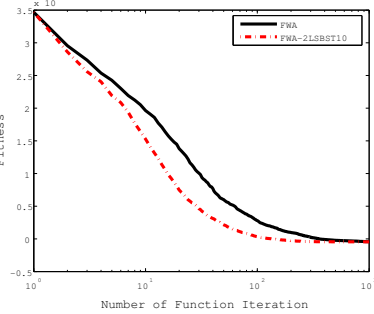

F1

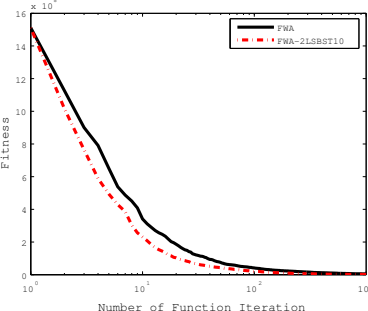

F3

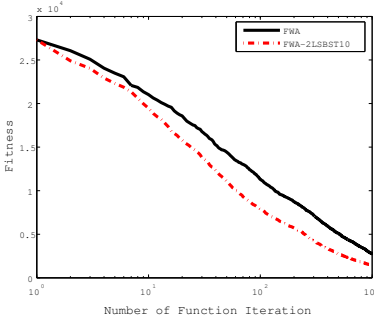

F5

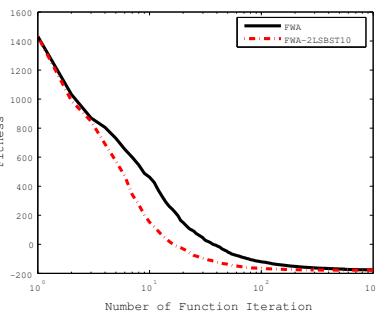

F7

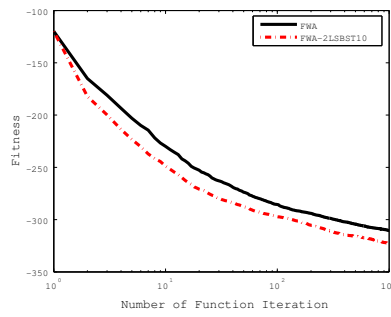

F9

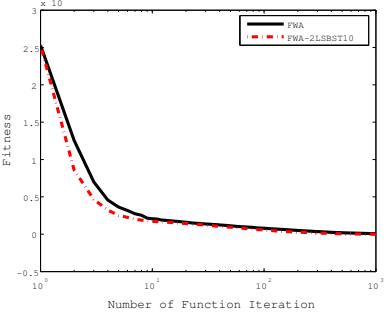

F2

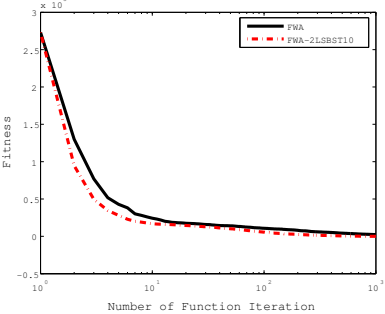

F4

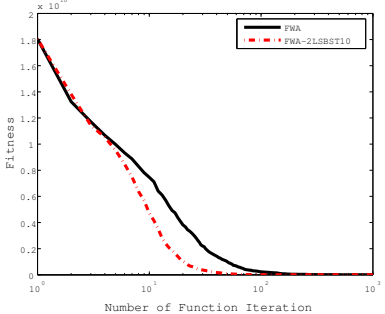

F6

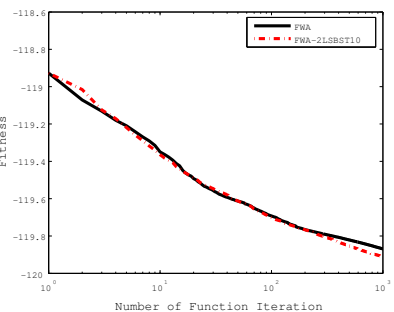

F8

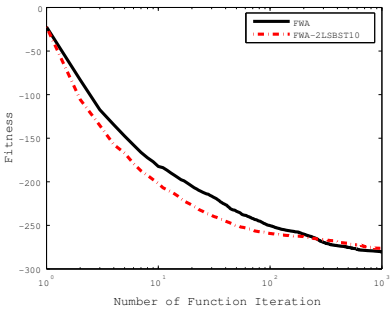

F10
Fig. 2. Average convergence curves of 50 trial runs for F1-F10, there are only the fireworks algorithm (FWA) and the the fireworks algorithm with elite strategy by LS2, best sampling method and 10 sampling number (FWA2LSBST10) in each sub figure. 
TABLE II

MEAN AND VARIANCE OF 10 BENCHMARK FUNCTIONS (THE VARIANCE IS PARENTHESIS) USED IN EXPERIMENTAL EVALUATIONS, THE BOLD FONT NUMBERS SHOW THE BETTER FINAL RESULTS.

\begin{tabular}{|c|c|c|c|c|c|c|c|c|c|c|}
\hline Methods & FT & $\mathrm{F} 2$ & F3 & F4 & F5 & F6 & F7 & F8 & F9 & F10 \\
\hline & $-4.27 \mathrm{E}+02(1.55 \mathrm{E}+03)$ & $6.61 \mathrm{E}+02(4.87 \mathrm{E}+05)$ & $4.77 \mathrm{E}+06(1.50 \mathrm{E}+13)$ & $2.36 \mathrm{E}+03(2.47 \mathrm{E}+06)$ & $2.77 \mathrm{E}+03(5.44 \mathrm{E}+06)$ & $4.64 \mathrm{E}+04(1.93 \mathrm{E}+10)$ & $-1.76 \mathrm{E}+02(2.43 \mathrm{E}+01)$ & $-1.20 \mathrm{E}+02(6.33 \mathrm{E}-03)$ & $-3.10 \mathrm{E}+02(1.20 \mathrm{E}+02)$ & $81 \mathrm{E}+02(3.12 \mathrm{E}+02)$ \\
\hline LS1-BST3 & $-4.16 \mathrm{E}+02(4.70 \mathrm{E}+03)$ & $7.37 \mathrm{E}+02(1.01 \mathrm{E}+06)$ & $4.04 \mathrm{E}+06(8.37 \mathrm{E}+12)$ & $2.68 \mathrm{E}+03(2.20 \mathrm{E}+06)$ & $3.46 \mathrm{E}+03(8.54 \mathrm{E}+0$ & $1.49 \mathrm{E}+05(1.03 \mathrm{E}+12)$ & $-1.76 \mathrm{E}+02(7.73 \mathrm{E}+01)$ & $-1.20 \mathrm{E}+02(9.62 \mathrm{E}-03)$ & $\mathrm{E}+02(1.12 \mathrm{E}+02)$ & \\
\hline & $-4.11 \mathrm{E}+02(3.13 \mathrm{E}+04)$ & $4.35 \mathrm{E}+02(6.06 \mathrm{E}+$ & $6(8.72 \mathrm{E}+12)$ & 2.13 & 2.9 & 3) & 0) & & & 2) \\
\hline BST10 & $-4.38 \mathrm{E}+0$ & $3.47 \mathrm{E}+02(4.61 \mathrm{E}$ & $5.21 \mathrm{E}+06(2.08$ & $2.23 \mathrm{E}+03$ & $3(7.2-2 y-3)$ & $2.05 \mathrm{E}+04(6.89 \mathrm{E}+09)$ & $2(1.21 \mathrm{E}+01)$ & & +02) & 02) \\
\hline BST3 & & & & & & & $2(1.12 \mathrm{E}+00)$ & 3) & & 2) \\
\hline & $-4.50 \mathrm{E}+02(1.26 \mathrm{E}-01)$ & $.00 \mathrm{E}+02(8.48 \mathrm{E}+04)$ & $1.56 \mathrm{E}+06(2.44 \mathrm{E}+12)$ & $7 \mathrm{E}+02(1.69 \mathrm{E}+05)$ & $2.20 \mathrm{E}+03(4.63 \mathrm{E}+\mathrm{C}$ & $1.62 \mathrm{E}+03(3.81 \mathrm{E}+06)$ & $-1.78 \mathrm{E}-2 \mathrm{C}-\mathrm{s}$ & 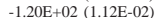 & & $+22(2)-2>1$ \\
\hline LS2-BST10 & $-4.50 \mathrm{E}+02(8.83 \mathrm{E}-$ & $22 \mathrm{E}-2 \mathrm{C}-2$ & (1.7 & 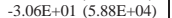 & $(2.21 \mathrm{~F}) \mathrm{F}$ & (6.23 & & -1. & & \\
\hline 53 & $-4.34 \mathrm{E}+02(4$. & $2(5.55 \mathrm{E}+0$ & $(1.03$ & $3(2.2-2>3$ & (6. & $1.36 \mathrm{~F}$ & & 2) & & \\
\hline LS1-DI & -4.12 & $28 \mathrm{E}+02(\mathrm{G}-2)-2$ & 3) & 2.12E $+03(2.49$ & $3(5.8$ & $4(1.25$ & $2(1$. & $-1.22-3=0$ & $12(1$ & 2) \\
\hline LS1-DIS10 & $-3.99 \mathrm{E}+02(4.2$ & $4.59 \mathrm{E}+02$ & $5.28 \mathrm{E}$ & 2.30 & 3.3 & $44(1.42-23$ & 11) & $-1.22-3>2$ & E+02) & 2) \\
\hline LS2-D & $-4.40 \mathrm{E}-2-2$ & $1.24 \mathrm{E}$ & $1.95 \mathrm{I}$ & 7.5 & & 3.5 & & & & \\
\hline LS2- & -4.41 & $-3.00 \mathrm{~F}$ & 1.51 & & 2.4 & & & & & \\
\hline & & -5.10 & & & & & & & & \\
\hline LS1-R & -3.831 & $7.81 \mathrm{E}$ & $4.86 \mathrm{E}$ & $2.22 \mathrm{E}+03$ & 2.9 & $1.93 \mathrm{E}+04(4.5$ & & -1.22 & 02) & $+02(2.97 \mathrm{E}+02)$ \\
\hline LS1-R & $-3.65 \mathrm{E}+02(1.4$ & $6.97 \mathrm{E}+02(8$. & $4.95 \mathrm{E}+06(2.78 \mathrm{E}+13)$ & $2.38 \mathrm{E}+03(2.49 \mathrm{E}$ & $2.37 \mathrm{E}+03(6.1$ & $7.05 \mathrm{E}+03(1.68 \mathrm{E}+08)$ & $-1.75 \mathrm{E}+02(3.14 \mathrm{E}+01)$ & $-1.20 \mathrm{E}+02(8.72 \mathrm{E}-03)$ & $-3.11 \mathrm{E}+02(9.83 \mathrm{E}+01)$ & $-2.80 \mathrm{E}+02(3.08 \mathrm{E}+02)$ \\
\hline LS1-RAN10 & $-3.79 \mathrm{E}+02(1.71 \mathrm{E}+05)$ & $5.69 \mathrm{E}+02(7.42 \mathrm{E}+05)$ & 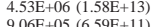 & $\begin{array}{l}2.45 \mathrm{E}+03(2.00 \mathrm{E}+06) \\
1.52 \mathrm{E}\end{array}$ & $3.24 \mathrm{E}+03(6.48 \mathrm{E}+06)$ & $5.11 \mathrm{E}+03(1.30 \mathrm{E}+08)$ & $-1.77 \mathrm{E}+02(8.99 \mathrm{E}+00)$ & $-1.20 \mathrm{E}+02(6.17 \mathrm{E}-03)$ & $-3.13 \mathrm{E}+02(1.36 \mathrm{E}+02)$ & $-2.79 \mathrm{E}+02(3.64 \mathrm{E}+02)$ \\
\hline $\begin{array}{l}\text { LS2-RAN3 } \\
\text { LS2-RAN5 }\end{array}$ & $\begin{array}{l}-4.50 \mathrm{E}+022(4.477 \mathrm{E}-02) \\
-4.50 \mathrm{E}+02(9.47 \mathrm{E}-02)\end{array}$ & $\begin{array}{l}-3.10 \mathrm{E}+202(9.48 \mathrm{E}+03) \\
-\mathbf{3 . 5 0 E + 0 2}(\mathbf{5 . 1 6 E}+\mathbf{0 3})\end{array}$ & $\begin{array}{l}9.06 \mathrm{E}+05(6.59 \mathrm{~g} \mathrm{E}+11) \\
9.47 \mathrm{E}+05 \\
(5.97 \mathrm{E}+11)\end{array}$ & $\begin{array}{l}-1.52 \mathrm{E}+02(3.922+04) \\
-2.51 \mathbf{0 0 2}(1.13 \mathbf{E}+04)\end{array}$ & $\begin{array}{l}1.84 \mathrm{E} \mathrm{E}+03(1.67 \mathrm{E}+06) \\
1.16 \mathrm{E}+03(1.11 \mathrm{E}+06)\end{array}$ & $\begin{array}{l}1.62 \mathrm{E} \mathrm{E}+03(4.14 \mathrm{EE}+06) \\
1.62+03(4.32 \mathrm{E}+06)\end{array}$ & $\begin{array}{l}-1.79 \mathrm{E}+02(6.28 \mathrm{E}-01) \\
-1.79 \mathrm{E}+02(4.34 \mathrm{E}-01)\end{array}$ & $\begin{array}{l}-1.20 \mathrm{E}+02(9.64 \mathrm{E}-03) \\
-1.20 \mathrm{E}+02(6.04 \mathrm{E}-03)\end{array}$ & $\begin{array}{l}02(5.12 \\
02(7.0)\end{array}$ & $\begin{array}{l}-2.88 \\
-2.90\end{array}$ \\
\hline S2-RAN 10 & $-4.47 \mathrm{E}+02(5.09 \mathrm{E}+00)$ & $-3.34 \mathrm{E}+02(3.51 \mathrm{E}+03)$ & $8.38 \mathrm{E}+05(2.93 \mathrm{E}+11)$ & $-2.43 \mathrm{E}+02(1.29 \mathrm{E}+04)$ & $-1.33 \mathrm{E}+02(2.12 \mathrm{E}+05)$ & $2.70 \mathrm{E}+03(8.18 \mathrm{E}+06)$ & $-1.78 \mathrm{E}+02(4.40 \mathrm{E}-01)$ & $-1.20 \mathrm{E}+02(6.75 \mathrm{E}-03)$ & $-3.15 \mathrm{E}+02(7.46 \mathrm{E}+01)$ & $-2.90 \mathrm{E}+02(3.64 \mathrm{E}+02)$ \\
\hline
\end{tabular}

TABLE I

TEN BENCHMARK FUNCTIONS USED IN EXPERIMENTAL EVALUATIONS FROM [10] (UNI=UNIMODAL, MUL=MULTIMODAL, SH=SHIFTED, $\mathrm{RT}=$ ROTATED, $\mathrm{GB}=\mathrm{GLOBAL}$ ON BOUNDS, NS=NON-SEPARABLE, AND $\mathrm{S}=$ SEPARABLE.

\begin{tabular}{|l|l|l|l|l|}
\hline No. & Name & Range & Optima & Characters \\
\hline F1 & Sh Sphere & {$[-100,100]$} & -450 & Sh-Uni-S \\
F2 & Sh Schwefel 1.2 & {$[-100,100]$} & -450 & Sh-Uni-NS \\
F3 & Sh Rt Elliptic & {$[-100,100]$} & -450 & Sh-Rt-Uni-NS \\
F4 & F2 with Noise & {$[-100,100]$} & -450 & Sh-Uni-NS \\
F5 & Schwefel 2.6 GB & {$[-100,100]$} & -310 & Uni-NS \\
F6 & Sh Rosenbrock & {$[-100,100]$} & 390 & Sh-Mul-NS \\
F7 & Sh Rt Griewank & {$[0,600]$} & -180 & Sh-Rt-Mul-NS \\
F8 & Sh Rt Ackley GB & {$[-32,32]$} & -140 & Sh-Rt-Mul-NS \\
F9 & Sh Rastrigin & {$[-5,5]$} & -330 & Sh-Mul-Sep \\
F10 & Sh Rt Rastrigin & {$[-5,5]$} & -330 & Sh-Rt-Mul-NS \\
\hline
\end{tabular}

\section{EXPERIMENTAL EVALUATIONS}

\section{A. Experimental Design}

To investigate the influence of sampling methods and sampling number on the acceleration performance, we used the fireworks algorithm as the optimization method to study our research proposals. Ten benchmark functions are selected as a test suite [10], Table I shows their range, optima and characteristic. In our experiments, we compare our proposed acceleration approaches by least squares approximation with a normal fireworks algorithm, conduct t-test for each proposed acceleration methods, of course, we checked normality of data distribution before applying t-test.

Here, we abbreviate the fireworks algorithm where the search space is regressed by a two-degree least square approximation as FWA-LS2, where it is regressed by a line power function least squares approximation as FWA-LS1. The best, distance near the best fitness individual and random sampling method are abbreviated as BST, DIS and RAN, followed by the sampling number. These abbreviations are also used in Figure 2, Table II, III and IV.

In this paper, the number of fireworks $(N)$ and Gaussian mutation firework $(\hat{m})$ are both set as 8 and other parameters are set as in Reference [12]. Experimental evaluations run 50 trails of 1000 generations on each ten benchmark functions independently. All the benchmark functions are set as 10 dimensions. The experimental platform for all evaluations is
MATLAB $R 2011 b$, based on an Intel(R) Core(TM) i7-2600 CPU, 4G RAM machine, and runs under Windows $(7)$.

\section{B. Experimental Results}

Figure 2 shows the convergence curve of each benchmark function. Table II shows the mean and variance of ten benchmark functions, and Table III shows the t-test results of each acceleration method compared to the normal fireworks algorithm.

From those experimental results, we can conclude that:

(1). Elite strategy is an effective approach to enhance fireworks algorithm search capability.

(2). The random sampling method has the better acceleration performance than the others.

(3). For some benchmark functions, best sampling method has the same acceleration performance as the random sampling method.

(4). The distance sampling method is not more effective than the other sampling methods, i.e best and random sampling method.

(5). In the distance sampling method, the approximation model by the more sampling number have the better performance than the other approaches, such as F9 and F10.

(6). For all the benchmark functions, LS2 methods are better LS1 methods.

\section{Discussion}

Table IV shows the confidence interval of the $p$ value for each approximation approach. We analyze the influence of approximation approaches from the experimental results of Tables II, III, IV and Figures 2.

\section{A. Fireworks Algorithm Acceleration Performance}

Fireworks algorithm is a new EC algorithm, which is inspired by the nature phenomenon of firework exploding. The crucial mechanism of fireworks algorithm is the multichange strategy, i.e. one firework makes several sparks for implementing the multi path searches in the optimization process, which is the principal difference from evolution strategy. This mechanism presents the fireworks algorithm optimization capability and originality. This kind of multi-change strategy can be applied to other ECs for obtaining the improved optimization performance, and this is our future work. 
TABLE III

T-TEST RESULTS OF 10 BENCHMARK FUNCTIONS USED IN EXPERIMENTAL EVALUATIONS, THE BOLD FONT MEANS THE SIGNIFICANCE OF THE PROPOSED ACCELERATION METHODS IN THE SIGNIFICANT LEVEL OF 0.05.

\begin{tabular}{|c|c|c|c|c|c|c|c|c|c|c|c|}
\hline Methods & F1 & F2 & F3 & F4 & F5 & F6 & F7 & F8 & F9 & F10 & Average \\
\hline LS1-BST3 & 0.307332147 & 0.662649583 & 0.290762839 & 0.2938901 & 0.194128978 & 0.481792344 & 0.879507493 & 0.359460163 & 0.911044864 & 0.501636817 & 0.488220533 \\
\hline LS1-BST5 & 29755856 & 0.128353922 & 0.666255931 & 0.430167086 & 0.774250951 & 0.065896191 & 0.200308462 & 0.710350997 & 0.220415229 & 0.415884048 & 0.414163867 \\
\hline LS1-BST10 & 80568249 & 0.024709114 & 03967477 & 0.688709876 & 0.202003046 & 0.262785218 & 0.493764665 & 307790179 & 0.743280761 & 0.212774404 & 0.362035299 \\
\hline LS2-BST3 & 0.000188728 & $1.72887 \mathrm{E}-09$ & $6.38234 \mathrm{E}-06$ & $1.55102 \mathrm{E}-11$ & 031350006 & 0.027317035 & 0.003412136 & .575529811 & $3.13628 \mathrm{E}-07$ & 0.661585597 & 0.129939001 \\
\hline LS2-BST5 & 0.000191076 & $1.01565 \mathrm{E}-09$ & $9.00847 \mathrm{E}-07$ & $4.66566 \mathrm{E}-13$ & 0.21305291 & 0.027361664 & 0.001990835 & 0.580236828 & $1.63901 \mathrm{E}-07$ & 0.702019593 & 0.152485397 \\
\hline LS2-BST10 & 0.000181421 & $4.68746 \mathrm{E}-09$ & $2.90968 \mathrm{E}-07$ & $\mathrm{E}-14$ & 0.000641905 & 0.028505937 & 0.001059495 & 0.007109283 & $5.25 \mathrm{E}$ & & 0.029837932 \\
\hline LS1-DIS3 & 0.30245976 & 0.092481313 & 0.210535165 & 0.733537611 & 0.291850925 & 0.034498433 & 0.958691195 & 0.16102296 & 0.562151761 & 0.286434974 & 0.36336641 \\
\hline LS1-DIS5 & 0.301933798 & 0.824931301 & 0.837812656 & 0.435099685 & 0.267385903 & 0.08340364 & 0.85807477 & 0.661467246 & 0.373860908 & 0.907668024 & 0.555163793 \\
\hline LS1-DIS10 & 0.356737538 & 0.149570418 & 0.598343293 & 0.859300138 & 0.265099553 & 0.102485067 & 0.252560319 & 0.134594188 & 0.836520872 & 0.154126974 & 0.370933836 \\
\hline LS2-DIS3 & 0.040201261 & $1.0228 \mathrm{E}-05$ & $\mathbf{9 . 0 7 7 1}$ & 5.572 & 0.258 & 0.034213051 & 0.012181648 & 0.508614684 & 0.216969334 & 0.06 & 0.113650887 \\
\hline LS2 & 157 & 4.103 & & & & & & & 37 & 499 & 4625 \\
\hline LS2-DIS10 & 0.024993346 & $3.36763 \mathrm{E}-08$ & $2.93311 \mathrm{E}-07$ & 2.7264 & 0.088265815 & 0.037 & 0.0004629 & 0.968829729 & 0.041298242 & 0.002334547 & 0.11 \\
\hline LS1 & 0.299 & 0.47. & & 0. & & & & & & & 347364 \\
\hline & & 0.827 & 0. & & 15 & 0. & 7 & 0.5 & 16 & 001 & 0.621 \\
\hline LS1-F & 2206 & 0. & & 55 & 29 & & & & & & 2008 \\
\hline LS2-RAN3 & 0166072 & $3.15916 \mathrm{E}$ & 6.190 & $2.4 / 88$ & 89 & 0.0273 & 0. & 0.19381 & $3.40565 \mathrm{E}-05$ & 0.47 & 0.071646357 \\
\hline LS2-RAN5 & 0183797 & $8.46373 \mathrm{E}-14$ & $7.99951 \mathrm{E}-09$ & $6.78613 \mathrm{E}-16$ & 3.2459 & 0.0273 & 19 & 0.9 & 0.000 & 781 & 0.099691522 \\
\hline LS2- & 4984 & 6E-13 & $63742 \mathrm{E}-09$ & E-16 & 1665 & 424 & 627 & 551 & 577 & 4202 & 2037 \\
\hline
\end{tabular}

It is one of the main proposals of this paper to obtain the fitness landscape to enhance fireworks algorithm performance, we use polynomial regression of one and two degree function as the approximation model. From the evaluation result, we can conclude that our proposed elite strategy by obtaining the fitness landscape is an effective method to enhance fireworks algorithm search capability. Most of our used sampling methods for approximating the fitness landscape in projected one-dimensional space with different sampling number can accelerate fireworks algorithm search.

\section{B. Approximation Methods}

We use one and two degree polynomial function as the approximation model to approximate fitness landscape into linear and nonlinear search space. Experimental results in Table III suggests that two degree model is more effective to accelerate the fireworks algorithm search than the one degree model in most of the benchmark functions. It is because that two degree model, i.e. nonlinear model can extend the search space range than the linear model, where the fireworks algorithm conducts a search within the limited search space.

Nonlinear models (LS2) are better approximating the fitness landscape for the ten benchmark functions than linear models (LS1) from Table IV. From our comparative evaluations, nonlinear model shows its strong capability to extend the potential global optima region in the search space. For most of the real world EC application, nonlinear models are the beneficial regression model to approximate the fitness landscape both in approximation model accuracy and acceleration performance.

\section{Sampling Methods}

In the experimental evaluations, we investigate three sampling methods, i.e. best sampling method, distance near the best fitness individual sampling method, random sampling methods. For the most of the benchmark functions, the acceleration performance with the best sampling method and random sampling are better than the distance sampling.

On the one hand, the spark with the better fitness locate in the global optima region, and the approximation model fitted by those data can obtain the accurate model for accelerating. On the other hand, the spark distance near the spark with
TABLE IV

CONFIDENCE INTERVAL OF P-VALUE OF APPROXIMATION METHODS, SAMPLING METHOD AND SAMPLING NUMBER METHOD, THE SAMPLING NUMBER FOR EACH METHOD IS 60, I.E. IT FOLLOWS A NORMAL DISTRIBUTION ACCORDING TO THE CENTRAL LIMIT THEOREM, AND THE CONFIDENT PROBABILITY IS 0.95, I.E. U $=1.96$

\begin{tabular}{|c|c|c|c|}
\hline Methods & Mean & Variance & Confident Interval \\
\hline LS1 & 0.455344875 & 0.077382406 & {$[0.435764425,0.474925325]$} \\
\hline LS2 & 0.10014382 & 0.046685584 & {$[0.088330737,0.111956903]$} \\
\hline$\overline{B S T}$ & 0.262780338 & 0.079985937 & {$[0.242541104,0.283019573]$} \\
\hline DIS & 0.274161028 & 0.0925 & {$[0.250747924,0.297574133]$} \\
\hline RAN & 0.296291675 & 0.110277386 & {$[0.268387647,0.324195704]$} \\
\hline Sampling \# 3 & 0.273445092 & 0.083 & {$[0.252273342,0.294616842]$} \\
\hline Sampling \# 5 & 0.328013328 & 0.114 & {$[0.299083552,0.356943105]$} \\
\hline Sampling \# 10 & 0.231774622 & 0.080643022 & {$[0.211369122,0.252180122]$} \\
\hline
\end{tabular}

the best fitness does not mean they are also close to the global optima region, and the approximation accuracy and acceleration performance by distance sampling are worse than that by the other sampling methods. From our comparative evaluation, we found those two points.

Sampling methods cannot take effect in isolation, which have a better acceleration performance only with the proper approximation methods. In our study, the proper approximation methods are the two degree polynomial model, i.e. nonlinear model.

Best sampling method can enhance fireworks algorithm search effectively for all the benchmark functions except F10.

Distance near the best fitness individual sampling method is effective in most of the cases, except F5, F8, and F10. However, it needs more computational cost in computing the distance, in the practical points of view, it is a useless sampling method.

The random sampling method can accelerate all the benchmark functions with nonlinear approximation model form Table III. The elite obtained by this method can increase the population diversity. This is the new discovery from our study work. Because of the random sampling method is with the less computational cost in the sampling process than best and distance sampling methods, which need more search and sort operations, it is the beneficial sampling method to the application of the proposed method in the practical points of view.

If the sampling data belong to a certain distribution, may 
be the acceleration performance can be improved with the concrete fitness landscape, the future research will involve this direction.

\section{Sampling Data Number}

The sampling data number is crucial for obtaining an accurate approximation model to accelerate the fireworks algorithm search. Theoretically, more number of sampling data means obtaining more accuracy approximation model and better acceleration performance.

From Table II, the acceleration performance by the best sampling with LS2 and five and ten sampling have better performance than that with three sampling data.

From Table III and Table IV, distance sampling method with 10 sampling data are effective in F1, F2, F3, F4, F6, F7, F9 and F10, it can show the relationship between sampling data number and acceleration performance, i.e. The more sampling number means more better approximation and acceleration performance.

Sampling data number decides the computational cost in the approximation regression computing, so we should select the proper sampling number to obtain approximation model by balancing the computational cost and acceleration performance for a concrete landscape or a real world application.

\section{CONCLUSION AND FUtURE WORK}

In this paper, we investigated a series of methods to enhance the fireworks algorithm by elite strategy. There are three sampling methods, which we used in our methods to approximate fireworks algorithm fitness landscape. We conduct a set of comparative evaluations to study on the sampling method and number influence on the approximation accuracy and acceleration performance. From our experimental evaluations, there are five discoveries in this research as follows.

(1) Our proposed elite strategy is an efficient method to enhance fireworks algorithm search capability significantly.

(2) Sampling method cannot take effect in isolation, it must be with a proper approximation model to accelerate the fireworks algorithm search for a certain benchmark function, i.e. fitness landscape.

(3) For some cases, the best sampling method and the random sampling method have the same acceleration performance.

(4) It can be obtained by a fitness landscape approximation accuracy and acceleration performance with the more sampling data number and a proper approximation model. In our study, the better approximation model is the nonlinear model (LS2).

(5) In the practical points of view, the random sampling method is a better tool to obtain the higher acceleration performance in both computational time and final solution quality.

Although those results come from just ten benchmark evaluations, the test times for each approximation model, sampling method and sampling number are up to 90 times, 60 times and 60 times, which belongs to the large sample analysis. According to the small probability principal, our conclusion is general and our empirical study can be as the reference to the real world application.
There are three remaining study topics. First, we use the elite to accelerate the fireworks algorithm, the obtained elite is whether the best individual in the population? In another word, the best firework reserved by fireworks algorithm is powerful or the elite obtained by landscape approximation is powerful? Second, in the approximation and the obtaining elite process, the elite from interpolation or extrapolation, which is more frequent? And how about their influence to the fireworks algorithm acceleration? Third, if we use different methods for approximating fitness landscape, i.e. interpolation, regression, neural networks, kernel based regression, etc. Which is more beneficial for approximation model accuracy and fireworks algorithm acceleration performance? We will conduct studies on those topics and answer those questions in the future.

\section{ACKNOWLEDGMENT}

This work was supported in part by Grant-in-Aid for Scientific Research (23500279) Japan and in part by the National Natural Science Foundation of China under grants number 61170057 and 60875080. Yan Pei would like to thank Yoshida Scholarship Foundation for its support of his doctoral research.

\section{REFERENCES}

[1] Andreas J. and Tan Y., "Swarm Intelligence for Non-negative Matrix Factorization", Int. J. of Swarm Intelligence Research, 2(4), pp.12-34, (Oct.-Dec., 2011).

[2] Andreas J. and Tan Y., "Iterative Improvement of the Multiplicative Update NMF Algorithm Using Nature-inspired Optimization”. 2011 Seventh Int. Conf. on Natural Computation (ICNC2011), pp.1668-1672, Shanghai, China (26-28, Jul. 2011).

[3] Jin, Yaochu, "A Comprehensive Survey of Fitness Approximation in Evolutionary Computation", Soft Computing, Vol.9, No.1, pp.3-12 (2005).

4] Jin, Yaochu, "Surrogate-assisted Evolutionary Computation: Recent Advances and Future Challenges", Swarm and Evolutionary Computation, 1(2), pp.61-70, (2011)

[5] Pei Y. and Takagi, H., "Accelerating Evolutionary Computation with Elite Obtained in Projected One-Dimensional Spaces", 5th Int. Conf. on Genetic and Evolutionary Computing (ICGEC2011), pp.89-92, Kimmen, Taiwan (Aug. 29 - Spt. 1, 2011)

[6] Pei Y. and Takagi H., "A Survey on Accelerating Evolutionary Computation Approaches", Third Int. Conf. on Soft Computing and Pattern Recognition (SoCPaR2011), pp.201-206, Dalian, China (14-16, Oct. 2011).

[7] Pei Y. and Takagi H., "Comparative Evaluations of Evolutionary Computation with Elite Obtained in Reduced Dimensional Spaces", Third Int. Conf. on Intelligent Networking and Collaborative Systems (INCoS2011), pp.35-40, Fukuoka, Japan (30, Nov. - 2, Dec. 2011).

[8] Pei Y. and Takagi H., "Fourier analysis of the Fitness Landscape for Evilutionary Search Acceleration", 2012 IEEE Congress on Evolutionary Computation (IEEE CEC 2012), pp.2934-2940, Brisbane, Australia (1015, Jun. 2012)

[9] Pei Y. and Takagi H., "Comparative Study on Fitness Landscape Approximation with Fourier Transform", 6th Int. Conf. on Genetic and Evolutionary Computing (ICGEC2012), Kitakyushu, Japan (25-28, Aug. 2012).

[10] Suganthan P., Hansen N., Liang J., Deb K., Chen Y., Auger A. and Tiwari S., "Problem Definitions and Evaluation Criteria for the CEC 2005 Special Session on Real-Parameter Optimization", Technical Report, Nanyang Technological University, Singapore, "http : //www.ntu.edu.sg/home/EPNSugan" (2005).

[11] Takagi H., Ingu T., and Ohnishi K., "Accelerating a GA Convergence by Fitting a Single-Peak Function", J. of Japan Society for Fuzzy Theory and Intelligent Informatics, vol.15, no.2, pp.219-229 (2003) (in Japanese).

[12] Tan Y. and Zhu Y. C., "Fireworks Algorithms for Optimization", Proc. of Int. Conf. on Swarm Intelligence (ICSI2010), Part II, LNCS 6145 , pp.355-364, Beijing, China (12-15, Jun. 2010). 